\title{
A Remark on Cauchy-Kowalevski's Theorem
}

\author{
By \\ Masatake MIYAKE*
}

\section{$\S 1$. Introduction}

Professor S. Mizohata has proposed in his paper [5]: what is a kowalevskian system? And he has given a necessary condition to hold the Cauchy-Kowalevski theorem for the general system of linear partial differential equations. Especially for the single equation,

$$
\partial_{t}^{m} u+\sum_{j=1}^{m} a_{j}\left(x, t ; \partial_{x}\right) \partial_{t}^{m-j} u=f,
$$

(where all the coefficients and $f$ are analytic in a domain $V \subset \boldsymbol{R}^{s+1}$ ), he has given the following result: in order that the Cauchy-Kowalevski theorem for (1.1) hold at every point in $V$, it is necessary and sufficient that the order of $a_{j}\left(x, t ; \partial_{x}\right) \leqq j$ in $V$. (see Th. 2 and its remark in [5])

We want to give a more strong characterization of the kowalevskian equation, but in this article we consider only the following equation,

$$
\partial_{\imath} u=\sum_{|\alpha| \leqq m} a_{\alpha}(x, t) \partial_{x}^{\alpha} u,
$$

where $a_{\alpha}(x, t) \in H(\Omega), \quad \Omega=\Omega_{x} \times \Omega_{t} \subset C^{s} \times C^{1}, \quad \Omega_{x}=\left\{x \in C^{s} ;|x|<r_{1}\right\}, \quad \Omega_{t}=$ $\left\{t \in C^{1} ;|t|<r_{2}\right\}$, and $H(\Omega)$ denotes the set of all holomorphic functions defined on $\Omega$. The topology of $H(\Omega)$ is given by the uniform convergence on every compact set in $\Omega$, and by its topology $H(\Omega)$ is a Fréchet space.

In order to give a precise statement of our theorem, let us give a definition.

Definition 1. We say that the Cauchy-Kowalevski theorem for (1.2)

Communicated by S. Matsuura, December 11, 1973.

* Department of Mathematics, University of Tsukuba, 300-31, Tsukuba. 
holds at a point $\left(x_{0}, t_{0}\right) \in \Omega$, if for any holomorphic function $u_{0}(x)$ in a neighborhood of $x=x_{0}$, there exists a unique holomorphic solution $u(x, t)$ of (1.2) in a neighborhood of $\left(x_{0}, t_{0}\right)$ satisfying $\left.u\right|_{t=t_{0}}=u_{0}(x)$.

Then we have the following

Theorem. The Cauchy-Kowalevski theorem for (1.2) holds at a point $\left(x_{0}, t_{0}\right) \in \Omega$ if and only if $a_{\alpha}(x, t) \equiv 0$ in $\Omega$ for any $\alpha$ such as $|\alpha| \geqq 2$.

Hence, the kowalevskian equation is best possible to consider the theorem of type Cauchy-Kowalevski.

The sufficiency is the classical Cauchy-Kowalevski theorem, so we show only the necessary condition. Without loss of generality we shall prove the necessity by setting $\left(x_{0}, t_{0}\right)=(0,0)$. When the case where $a_{\alpha_{0}}(0,0) \neq 0$ for some $\alpha_{0}\left(\left|\alpha_{0}\right|=m \geqq 2\right)$, trivially the Cauchy-Kowalevski theorem does not hold at the origin, therefore we must prove that when $a_{\alpha}(0,0)=0, a_{\alpha}(x, t) \not \equiv 0$ for some $\alpha(|\alpha| \geqq 2)$, the Cauchy-Kowalevski theorem does not hold at the origin. Thus, let us decompose the coefficient $a_{\alpha}(x, t)$ in (1.2) as follows,

$$
a_{\alpha}(x, t)=a_{\alpha}(x) t^{n_{\alpha}}+b_{\alpha}(x, t) t^{n_{\alpha}+1},
$$

$n_{\alpha} \geqq 0$ (integer) and $a_{\alpha}(x) \not \equiv 0$, and we rewrite the equation (1.2) followingly,

$$
\partial_{t} u=\sum_{|\alpha| \leqq m}\left\{a_{\alpha}(x) t^{n_{\alpha}}+b_{\alpha}(x, t) t^{n_{\alpha}+1}\right\} \partial_{x}^{\alpha} u
$$

Now let us define modified order of differential operator.

Definition 2.1) We say that the modified order of $a_{\alpha}(x) t^{n_{\alpha}} \partial_{x}^{\alpha}$ at $t=0$ is $\frac{|\alpha|}{n_{\alpha}+1}$. We say that $\sum_{|\alpha|=j_{0}} a_{\alpha}(x) t^{n_{\alpha}} \partial_{x}^{\alpha}\left(n_{\alpha}=\right.$ const.) is a modified principal part of (1.3) at $t=0$, if $\frac{j_{0}}{n_{\alpha}+1}=\max _{\alpha}\left\{\frac{|\alpha|}{n_{\alpha}+1}\right\}$ and if $\frac{j_{0}}{n_{\alpha}+1}>\max _{|\alpha|>j_{0}}$ $\left\{\frac{|\alpha|}{n_{\alpha}+1}\right\}$.

1) This notion will be used in another problems, for instance, in the degenerate parabolic differential equations. (see M. Miyake [3], [4]). 
An elementary lemma for the proof of our theorem is the following one which is a special case of our theorem.

Lemma 1. If the modified principal part of (1.3) at $t=0$ consists of the part of $|\alpha| \geqq 2$, then there exists a point $x_{0} \in \Omega_{x}$ such that the Cauchy-Kowalevski theorem does not hold at $\left(x_{0}, 0\right)$. And the set of such a point $x_{0} \in \Omega_{x}$ is dense in $\Omega_{x}$. More precisely, we can construct a holomorphic function $u_{0}(x) \in H\left(\Omega_{x}\right)$ such that there can not exist holomorphic solution of (1.3) satisfying $\left.u\right|_{t=0}=u_{0}(x)$ in any neighborhood of $\left(x_{0}, 0\right)$.

Our method of the proof can not be applied to the equation (1.1). Seemingly similar result should be obtained for the equation (1.1), but its proof will be fairly complicated.

In section 2 we prove the theorem, in section 3 we investigate properties of modified order given in Definition 2 and from section 4 on we prove Lemma 1.

\section{§2. Proof of the Theorem}

In this section we prove our theorem using Lemma 1, and we provide the following two lemmas. Now let us rewrite the equation (1.2) as follows,

$$
\left[\partial_{t}+\sum_{j=1}^{s} a_{e_{j}}(x, t) \partial_{x_{J}}\right] u=\sum_{2 \leqq|\alpha| \leqq m} a_{\alpha}(x, t) \partial_{x}^{\alpha} u+a(x, t) u,
$$

where $e_{j}=\left(0, . ., 0, \frac{1}{j}, 0, \ldots, 0\right), a_{e_{j}}, a_{\alpha}, a \in H(\Omega)$. Then we have

Lemma 2. For any positive integer $l$, there exists a holomorphic transformation of the coordinates $(x, t) \rightarrow(y, t)$ having the following properties in a neighborhood of the origin;

(i) By its transformation, the equation (2.1) is transformed to

$$
\left[\partial_{t}+\sum_{j=1}^{s} t^{l} b_{e_{j}}(y, t) \partial_{y_{j}}\right] u=\sum_{|\alpha| \leqq m} b_{\alpha}(y, t) \partial_{y}^{\alpha} u
$$

(ii) In the right hand side of (i), the modified principal part at $t=0$ is that of the right hand side of (2.1). 
Remark. Lemma 2 asserts that when $a_{\alpha}(x, t) \not \equiv 0 \quad(|\alpha| \geqq 2)$, there exists such a transformation of the coordinates that by its transformation modified principal part at $t=0$ consists of the part of $|\alpha| \geqq 2$.

Proof. It suffices to prove the lemma when $l=1$, since for general $l(\geqq 2)$ we can prove by induction on $l$. Let $y_{j}(t ; x)(j=1, \ldots, s)$ be a solution of $\frac{d y_{j}}{d t}+a_{e_{j}}(y, t)=0(j=1, \ldots, s)$ satisfying $y_{j}(0 ; x)=x_{j}(j=$ $1, \ldots, s)$, and let the transformation of the coordinates $((x, t) \rightarrow(y, t))$ be $y_{j}=y_{j}(t ; x)(j=1, \ldots, s)$, then it is obvious that

$$
\begin{aligned}
& \partial_{t} \longrightarrow \partial_{t}-\sum_{j=1}^{s} a_{e_{j}}(y(t ; x), t) \partial_{y_{J}}, \\
& \partial_{x_{J}} \longrightarrow \partial_{y_{j}}+t \sum_{k=1}^{s} c_{j k}(x, t) \partial_{y_{k}},
\end{aligned}
$$

since $\frac{\partial y_{j}}{\partial t}=\frac{d y_{j}}{d t}$ and $\left.\frac{\partial y_{j}}{\partial x_{k}}\right|_{t=0}=\delta_{j k}$, where $\delta_{j k}$ is Kronecker's $\delta$. Thus we have that $\partial_{t}+\sum_{j} a_{e_{j}}(x, t) \partial_{x_{j}} \rightarrow \partial_{t}+\sum_{j} c_{e_{j}}(x, t) \partial_{y_{j}}$ where $c_{e_{j}}(x, t)=a_{e_{j}}(x, t)$ $-a_{e_{j}}(y(t ; x), t)+\sum_{k} t a_{e_{k}} c_{k j}$. Considering that $y_{j}(0 ; x)=x_{j}$, we have

$$
\partial_{t}+\sum_{j} a_{e_{j}}(x, t) \partial_{x_{j}} \longrightarrow \partial_{t}+t \sum_{j} b_{e_{j}}(y, t) \partial_{y_{j}} .
$$

On the other hand it is obvious that

$$
\partial_{x}^{\alpha} \longrightarrow \partial_{y}^{\alpha}+t \sum_{|\beta| \leqq|\alpha|} b_{\beta}(y, t) \partial_{y}^{\beta}
$$

Hence, in the right hand side of (i) its modified principal part at $t=0$ is that of the right hand side of (2.1). Finalyl we note that if $\left.\partial_{t}^{j} a(x, t)\right|_{t=0}$ $\equiv 0(j=0,1, \ldots, l-1)$ then it follows that $\left.\hat{o}_{t}^{j}\{a(x, t)-a(y(t ; x), t)\}\right|_{t=0} \equiv 0$ $(j=0,1, \ldots, l)$. From this the lemma follows for general $l \geqq l$. Q.E.D.

Generally, the next lemma is obtained from Baire's category theorem.

Lemma 3. If for (1.2) the Cauchy-Kowalevski theorem holds at the origin, then there exists a domain $D_{\delta}=\left\{(x, t) \in C^{s} \times C^{1} ;|x|<\delta\right.$, $|t|<\delta\}$ ( $\delta$ is a positive constant) such that for any $u_{0}(x) \in H\left(\Omega_{x}\right)$ there exists a unique solution $u(x, t) \in H\left(D_{\delta}\right)$ of (1.2) with the Cauchy datum $u\left(x_{0}\right)$. 
For the proof see Proposition 1 in [5], or we can prove it by the similar way with Proposition 4.1 in [6] since the uniqueness of the solution (if it exists) follows in view of the construction of the formal solution $u(x, t) \sim \sum_{m \geq 0} u_{m}(x) t^{m} / m$ !, where $u_{0}(x)$ is the Cauchy datum.

Using above lemmas we can prove our theorem.

Proof of the Theorem. We prove it by the principle of contradiction. So we assume that $a_{x}(x, t) \not \equiv 0$ for some $|\alpha| \geqq 2$. We may assume that the modified principal part of (1.2) at $t=0$ consists of the part $|\alpha| \geqq 2$ from Lemma 2. If the Cauchy-Kowalevski theorem holds at the origin, then by Lemma 3 there exists a common existence domain $D_{\delta}$ of the solution for any Cauchy datum $u_{0}(x) \in H\left(\Omega_{x}\right)$. Let $x_{0} \in D_{\delta} \cap\{t=0\}$ be a point stated in Lemma 1, so there exists $u_{0}(x) \in H\left(\Omega_{x}\right)$ such that there can not exist holomorphic solution in any neighborhood of $\left(x_{0}, 0\right)$ with the Cauchy datum $u_{0}(x)$. This contradicts from the assumption of the solvability, because of $\left(x_{0}, 0\right) \in D_{\delta}$.

Q.E.D.

\section{§. Modified Order}

In this section we investigate modified order defined in section 1, and the main result is Lemma 4 . In order to justify the definition of modified order, we give a order relation between differential operators. Let

$$
\mathscr{L}_{i}=a_{i}(x) t^{n_{i}} \partial_{x}^{\alpha_{1}}, \quad n_{i} \geqq 0 \text { (integer), } a_{i}(x) \not \equiv 0,
$$

$(i=1,2)$ be two differential operators, then we give

Definition 3. We say that $\mathscr{L}_{1}>\mathscr{L}_{2}$ at $t=0$ if $\left|\alpha_{1}\right| /\left(n_{1}+1\right)>\left|\alpha_{2}\right| \mid$ $\left(n_{2}+1\right)$ and $\mathscr{L}_{1} \sim \mathscr{L}_{2}$ at $t=0$ if $\left|\alpha_{1}\right| /\left(n_{1}+1\right)=\left|\alpha_{2}\right| /\left(n_{2}+1\right)$.

For the differential operator $\mathscr{L}\left(x, t ; \partial_{x}\right)$ given by

$$
\mathscr{L}\left(x, t ; \partial_{x}\right)=\sum_{j=1}^{\text {finite }}\left\{a_{j}(x) t^{n_{J}}+\tilde{a}_{j}(x, t) t^{n_{J}+1}\right\} \partial_{x}^{\alpha_{j}},
$$

where $a_{j}(x) \not \equiv 0, n_{j} \geqq 0$ (integer), we rewrite it followingly, 


$$
\begin{aligned}
& \mathscr{L}=\sum_{j=1}^{k} \underbrace{t^{n_{J}} P_{j}\left(x ; \partial_{x}\right)}+\underbrace{\tilde{\mathscr{L}}\left(x, t ; \partial_{x}\right)}, \\
& \text { the part where the parts where modified } \\
& \text { modified order order are less than that } \\
& \text { is maximum. of } t^{n_{j}} P_{j} \text {. }
\end{aligned}
$$

where $P_{j}\left(x ; \partial_{x}\right)=\sum_{|\alpha|=p_{j}} a_{\alpha}(x) \partial_{x}^{\alpha}$. Without loss of generality we may assume that $0 \leqq n_{1}<n_{2}<\cdots<n_{k}$ and $0<p_{1}<p_{2}<\cdots<p_{k}$. In the decomposition (3.1), $\frac{p_{1}}{n_{1}+1}=\frac{p_{2}}{n_{2}+1}=\cdots=\frac{p_{k}}{n_{k}+1}$. We note also that the modified principal part of (3.1) at $t=0$ is $t^{n_{k}} P_{k}\left(x ; \partial_{x}\right)$ from Definition 2.

we have

Remark. If we rewrite $\mathscr{L}$ by $\mathscr{L}=\sum_{j \geqq 0} t_{j} \mathscr{L}_{j}\left(x ; \partial_{x}\right)$ instead of (3.1),

(i) order $\mathscr{L}_{j}<\frac{j+1}{n_{1}+1} p_{1} \quad$ if $j \neq n_{i}$,

(ii) order $\mathscr{L}_{n_{i}}=\frac{n_{i}+1}{n_{1}+1} p_{1}=p_{i}$ and

$\mathscr{L}_{n_{i}}=P_{i}+($ lower order terms), $\quad i=1,2, \ldots, k$,

where order $\mathscr{L}_{j}=\max _{x_{0} \in \Omega}\left\{\operatorname{order} \mathscr{L}_{j}\left(x_{0} ; \partial_{x}\right)\right\}$.

Let us consider the following Cauchy problem,

$$
\begin{aligned}
& \partial_{t} u=\mathscr{L}\left(x, t ; \partial_{x}\right) u, \\
& \left.u\right|_{t=0}=u_{0}(x) \in H\left(\Omega_{x}\right),
\end{aligned}
$$

where $\mathscr{L}$ is the differential operator given by (3.1). And let

$$
u(x, t) \sim \sum_{m \geqq 0} u_{m}(x) t^{m} / m !
$$

be a formal solution of the Cauchy problem (3.3)-(3.4), then $u_{m}(x)$ $(m \geqq 1)$ is represented by

$$
u_{m}(x)=S_{m}\left(x ; \partial_{x}\right) u_{0}(x), \quad(m \geqq 1),
$$

for some differential opertor $S_{m}\left(x ; \partial_{x}\right)$. In fact, $u_{m}(x)$ is given by substituting (3.5) into (3.3) and comparing the coefficients of $t^{m-1}$. Then for $S_{m}\left(x ; \partial_{x}\right)$ we have 
Lemma 4. (i) For $m=\sum_{i=1}^{k} l_{i}\left(n_{i}+1\right), l_{i} \geqq 0$ (integer) we have

$$
\text { order } S_{m}\left(x ; \partial_{x}\right) \leqq \frac{m p_{i}}{n_{i}+1}, \quad(i=1, \ldots, k)
$$

If we denote the principal part of $S_{m}$ by $Q_{m}\left(x ; \partial_{x}\right)$ (the part where the equality hold) we have

$$
Q_{m}(x ; \zeta)=\sum_{i=1}^{k} \frac{m !}{m \cdot\left(m-n_{i}-1\right) !} P_{i}(x ; \zeta) Q_{m-n_{i}-1}(x ; \zeta)
$$

$\left(\zeta \in C^{s}\right)$, where $Q_{m-n_{i}-1} \equiv 0 \quad$ if $\quad m-n_{i}-1 \neq \sum_{j=1}^{k} \tilde{l}_{j}\left(n_{j}+1\right) \quad$ for $\quad$ any $\quad \tilde{l}_{j} \geqq 0$ (integer), and $Q_{0}=1$.

(ii) For $m \neq \sum_{i=1}^{k} l_{i}\left(n_{i}+1\right)$ for any $l_{i} \geqq 0$ (integer), we have

$$
\text { order } S_{m}<\frac{m \cdot p_{i}}{n_{i}+1}
$$

Proof. Let $u(x, t) \sim \sum_{m \geqq 0} u_{m}(x) t^{m} / m$ ! be a formal solution of (3.3)(3.4), then obviously

$$
u_{m}(x) /(m-1) !=\sum_{j=0}^{m-1} \mathscr{L}_{j} u_{m-j-1}(x) /(m-j-1) !
$$

Therefore if we put $u_{m}(x)=S_{m}\left(x ; \partial_{x}\right) u_{0}(x)$, it follows that

$$
S_{m}\left(x ; \partial_{x}\right)=\frac{m !}{m}\left\{\sum_{j=0}^{m-1} \mathscr{L}_{j} S_{m-j-1} /(m-j-1) !\right\},
$$

where $S_{0}=1$.

The lemma is trivial for $m=1$, since $u_{1}(x)=\mathscr{L}_{0}\left(x ; \partial_{x}\right) u_{0}(x)$. In the case where $n_{1}>0$, we can prove that order $S_{m}<m p_{1} /\left(n_{1}+1\right)$ for $0<$ $m \leqq n_{1}$, by induction on $m$. In fact, if it is valid for $m=1, \ldots, m_{0}$ $\left(\leqq n_{1}-1\right)$, then considering $S_{m_{0}+1}=\frac{\left(m_{0}+1\right) !}{m_{0}+1}\left\{\sum_{j=0}^{m_{0}} \mathscr{L}_{j} S_{m_{0}-j} /\left(m_{0}-j\right) !\right\}$ we have that order $\mathscr{L}_{j} S_{m_{0}-j}<\left(m_{0}+1\right) p_{1} /\left(n_{1}+1\right)$. This implies order $S_{m_{0}+1}<$ $\left(m_{0}+1\right) p_{1} /\left(n_{1}+1\right)$.

Next, we consider $S_{n_{1}+1}=\frac{\left(n_{1}+1\right) !}{n_{1}+1} \mathscr{L}_{n_{1}}+\sum_{j=0}^{n_{1}-1}$ const. $\mathscr{L}_{j} S_{n_{1}-j}$. Considering $\mathscr{L}_{n_{1}}=P_{1}+($ lower order terms $)$ and order $\mathscr{L}_{j} S_{n_{1}-j}<p_{1}(j=0$, 
$\left.1, \ldots, n_{1}-1\right)$, we have $S_{n_{1}+1}=\frac{\left(n_{1}+1\right) !}{n_{1}+1} P_{1}+R_{n_{1}+1}$ where order $R_{n_{1}+1}<p_{1}$.

When $k=1$, we can prove the lemma by induction on $m$.

(i) $m \neq l\left(n_{1}+1\right)$ for any $l>1$ (intcger): let us consider (3.9). In the case where $j=n_{1}$, order $\mathscr{L}_{n_{1}}=p_{1}$. On the other hand, order $S_{m-n_{1}-1}$ $<\left(m-n_{1}-1\right) p_{1} /\left(n_{1}+1\right)$, since $m-n_{1}-1 \neq \tilde{l}\left(n_{1}+1\right)$ for any $\tilde{l} \geqq 0$ (integer). Hence, order $\mathscr{L}_{n_{1}} S_{m-n_{1}-1}<m p_{1} /\left(n_{1}+1\right)$. In the case where $j \neq n_{1}$, it is also valid that order $\mathscr{L}_{j} S_{m-j-1}<m p_{1} /\left(n_{1}+1\right)$, because of order $\mathscr{L}_{j}<(j+1) p_{1} /\left(n_{1}+1\right)$.

(ii) $m=l\left(n_{1}+1\right)$ for some $l \geqq 2$ (integer): in the case where $j=n_{1}$, $S_{m-n_{1}-1}=Q_{(l-1)\left(n_{1}+1\right)}+R_{(l-1)\left(n_{1}+1\right)}$ from the induction. Hence, $\mathscr{L}_{n_{1}} S_{m-n_{1}-1}$ $=P_{1} \cdot Q_{(l-1)\left(n_{1}+1\right)}+($ lower order terms). Finally, in the case where $j \neq n_{1}$, it follows that order $\mathscr{L}_{j} S_{m-j-1}<m p_{1} /\left(n_{1}+1\right)$.

This completes the proof in the case where $k=1$, and in this case $Q_{l\left(n_{1}+1\right)}$ is given explicitly by

$$
Q_{l\left(n_{1}+1\right)}(x ; \zeta)=\frac{\left\{l\left(n_{1}+1\right)\right\} !}{\left(n_{1}+1\right)^{l} \cdot l !}\left\{P_{1}(x ; \zeta)\right\}^{l}, \quad \zeta \in \mathbb{C}^{s} .
$$

In general case where $k \geqq 2$, we can also prove the lemma by induction on $m$. In fact, in view of (3.9) it suffices to consider $\mathscr{L}_{j} S_{m-j-1}$.

(iii) $m \neq \sum_{i=1}^{k} l_{i}\left(n_{i}+1\right)$ for any $l_{i} \geqq 0$ (integer): By the similar way with (i), it follows that order $\mathscr{L}_{j} S_{m-j-1}<m p_{i} /\left(n_{i}+1\right)(i=1, \ldots, k)$ from the induction on $m$.

(iv) $m=\sum_{i=1}^{k} l_{i}\left(n_{i}+1\right)$ for some $l_{i} \geqq 0$ (integer): in the case where $j \neq n_{i}(i=1,2, \ldots, k)$, order $\mathscr{L}_{j} S_{m-j-1}<m p_{i} /\left(n_{i}+1\right)(i=1, \ldots, k)$. In the case where $j=n_{i_{0}}$ for some $i_{0}, m-n_{i_{0}}-1=\sum_{i} \tilde{l}_{i}\left(n_{i}+1\right)$ for some $\tilde{l}_{i} \geqq 0$ or not for any $\tilde{l}_{i} \geqq 0$. In the latter case, order $\mathscr{L}_{n_{i_{0}}} S_{m-n_{i_{0}}-1}<m p_{i} /\left(n_{i}+1\right)$, but in the former case order $\mathscr{L}_{n_{i_{0}}} S_{m-n_{i_{0}}-1}=m p_{i} /\left(n_{i}+1\right)$ and its principal part is that of $P_{i_{0}}\left(x ; \partial_{x}\right) Q_{m-n_{i_{0}}-1}\left(x ; \partial_{x}\right)$.

This completes the proof.

Q.E.D.

The above lemma shows that the principal part $Q_{m}$ depends only on $P_{i}\left(x ; \partial_{x}\right)(i=1, \ldots, k)$ appeared in (3.1). We note that when $m=$ $\sum_{j} l_{j}\left(n_{j}+1\right), \quad m \cdot p_{i} /\left(n_{i}+1\right)=\sum_{j} l_{j} p_{j}$ since $p_{1} /\left(n_{1}+1\right)=\cdots=p_{k} /\left(n_{k}+1\right)$.

Now if we put $S_{m}\left(x ; \partial_{x}\right)=Q_{m}\left(x ; \partial_{x}\right)+R_{m}\left(x ; \partial_{x}\right)$, where order $R_{m}<$ $m p_{i} /\left(n_{i}+1\right)$, we have 


$$
u_{m}(x)=Q_{m}\left(x ; \hat{c}_{x}\right) u_{0}(x)+R_{m}\left(x ; \partial_{x}\right) u_{0}(x), \quad(m \geqq 1)
$$

Concerning $Q_{m}$ we have easily

Lemma 5. If $P_{i}\left(x_{0} ; \partial_{x}\right) \neq 0$ for some $i \in\{1,2, \ldots, k\}$, and some $x_{0} \in \Omega_{x}$, then for infinitely many $m Q_{m}\left(x_{0} ; \partial_{x}\right) \neq 0$.

Proof. We assume that $Q_{m}\left(x_{0} ; \partial_{x}\right) \neq 0$ for finite number of $m$ 's. We note also that for at least one $m, Q_{m}\left(x_{0} ; \partial_{x}\right) \neq 0$. Let $m_{0}=\sum_{i} \tilde{l}_{i}\left(n_{i}+1\right)$ (for some $\tilde{l}_{i} \geqq 0$ ) be a maximum such that $Q_{m_{0}}\left(x_{0} ; \partial_{x}\right) \neq 0$, and let $j_{0}=$ $\max \left\{j ; P_{j}\left(x_{0} ; \partial_{x}\right) \neq 0\right\}$. Then by (3.7) obviously we have that $Q_{m_{0}+n_{j 0}+1}$ $\left(x_{0} ; \partial_{x}\right)=c\left(j_{0}\right) P_{j_{0}}\left(x_{0} ; \partial_{x}\right) Q_{m_{0}}\left(x_{0} ; \partial_{x}\right) \neq 0$, where $c\left(j_{0}\right)$ is a constant appeared in (3.7). This contradicts from the determination of $m_{0}$.

Q.E.D.

\section{§4. Asymptotic Formula}

In this section, we consider a series $\left\{a_{m} ; m=\sum_{i=1}^{k} l_{i}\left(n_{i}+1\right)\right.$, for some non-negative integer $\left.l_{i}\right\}$ satisfying

$$
a_{m}=\sum_{j=1}^{k} \frac{m !}{m \cdot\left(m-n_{j}-1\right) !} c_{j} \cdot a_{m-n_{j}-1}, \quad c_{j} \neq 0 \in \mathbb{C},
$$

where $0 \leqq n_{1}<n_{2}<\cdots<n_{k}$. In the above formula, $a_{m-n_{1}-1}=0$ when $m-n_{j}-1 \neq \sum_{i} l_{i}\left(n_{i}+1\right)$ for any $l_{i} \geqq 0$ (integer) and $a_{0}=1$. We have easily that $a_{m} \neq 0$ for infinitely many $m$. From the proof of Lemma 5 we see that there exists at least one $m \in\left(m_{0}, m_{0}+n_{k}+1\right]$ such that $a_{m} \neq 0$ if $a_{m_{0}} \neq 0$.

Now let $a_{m_{0}} \neq 0$ and let $m(j)=m_{0}+n_{k}-n_{j}(j=1, \ldots, k)$ and $m(0)=$ $m_{0}+n_{k}+1$, then at least one of the following inequalities holds;

$$
\begin{aligned}
& \left|a_{m(j)} / a_{m(k)}\right| \geqq c \cdot m(j) ! / m(k) ! \quad(j=1, \ldots, k-1), \\
& \left|a_{m(0)} / a_{m(k)}\right| \geqq c \cdot m(0) ! / m(0) \cdot m(k) !,
\end{aligned}
$$

where $c=\min \left\{\frac{1}{k} \frac{\left|c_{k}\right|}{\left|c_{j}\right|}, \frac{\left|c_{k}\right|}{k} ; j=1, \ldots, k-1\right\}$.

In fact, if (4.2) does not hold, then obviously (4.3) holds.

We assume that corresponding with $\left\{n_{i}\right\}_{i=1}^{k}$ there exist positive 
integers $\left\{p_{i}\right\}_{i=1}^{k}$ satisfying

$$
\frac{p_{1}}{n_{1}+1}=\frac{p_{2}}{n_{2}+1}=\cdots=\frac{p_{k}}{n_{k}+1}, \quad 1 \leqq p_{1}<p_{2}<\cdots<p_{k}
$$

where $p_{k} \geqq 2$.

Our purpose in the followings is to show

$$
\varlimsup_{m \rightarrow \infty} \sqrt[m]{\left|a_{m}\right| \cdot\left\{\frac{m}{n_{k}+1} \cdot p_{k}\right\} ! / m !}=+\infty
$$

Now let $\{n(i)\}_{i=0}^{\infty}$ be a subsequence of $\left\{m ; m=\sum_{i} l_{i}\left(n_{i}+1\right)\right\}$ satisfying;

$n(0)=m_{0}=\min \left\{m ; a_{m} \neq 0\right\}$.

$n(1)$ is a minimum $m$ satisfying (4.2) or (4.3) when we set $m_{0}=$ $n(0)$. Generally, $n(i+1)$ is a minimum $m$ satisfying (4.2) or (4.3) when we set $m_{0}=n(i)$.

Considering that $a_{n(i)}=\frac{a_{n(i)}}{a_{n(i-1)}} \cdot \frac{a_{n(i-1)}}{a_{n(i-2)}} \ldots \cdot \frac{a_{n(1)}}{a_{n(0)}} \cdot a_{n(0)}$, therefore if $n(i)-n(i-1)<n_{k}+1$ for any $i=1,2, \ldots$, then by (4.2) we have

$$
\left|a_{n(i)}\right| \geqq A c^{i} \times n(i) !, \quad A=\left|a_{n(0)}\right| / n(0) ! .
$$

Generally from (4.2) and (4.3) we have

$$
\left|a_{n(i)}\right| \geqq A c^{i} \times n(i) ! / n(\sigma(1)) \cdot n(\sigma(2)) \cdot \cdots \cdot n\left(\sigma\left(m_{i}\right)\right),
$$

where $n(\sigma(1))<n(\sigma(2))<\cdots$ are defined by $n(\sigma(j))-n(\sigma(j)-1)=n_{k}+1$.

Obviously, $m_{i} \leqq\left[\frac{n(i)-n(0)}{n_{k}+1}\right]$ where [] denotes Gauss' symbol. On the other hand, since $n(0)+\left(k_{j}-1\right)\left(n_{k}+1\right) \leqq n(\sigma(j))<n(0)+k_{j}\left(n_{k}+1\right)$ for some $k_{j} \geqq 2$, we have

$$
n(\sigma(j))<d\left(k_{j}-1\right)\left(n_{k}+1\right), \quad d \geqq \frac{n(0)}{n_{k}+1}+2 .
$$

Therefore it follows

$$
\begin{aligned}
\left|a_{n(i)}\right| & \geqq A \frac{c^{i}}{\left\{d\left(n_{k}+1\right)\right\}^{m_{i}}} \cdot \frac{n(i) !}{\left(k_{1}-1\right)\left(k_{2}-1\right) \cdots\left(k_{m_{i}}-1\right)} \\
& \geqq A \frac{c^{i}}{\left\{d\left(n_{k}+1\right)\right\}^{m_{i}}} \cdot \frac{n(i) !}{\left\lceil\frac{n(i)}{n_{k}+1}\right] !},
\end{aligned}
$$


in view of $2 \leqq k_{1}<k_{2}<\cdots<k_{m_{\imath}} \leqq\left[\frac{n(i)-n(0)}{n_{k}+1}\right]+1$. Hence we have

\section{Lemma 6.}

$$
\sqrt[n(i)]{\left|a_{n(i)}\right| \cdot\left\{\frac{n(i)}{n_{k}+1} \cdot p_{k}\right\} ! / n(i) !} \longrightarrow \infty \quad \text { as } i \longrightarrow \infty
$$

Proof. Considering (4.7) and the assumption $p_{k} \geqq 2$, (4.8) is obvious.

\section{§5. Proof of Lemma 1}

From the arguments in section 3, we may consider the following Cauchy problem;

$$
\begin{aligned}
& \partial_{t} u=[\sum_{j=1}^{k} \underbrace{\boldsymbol{n}_{j} P_{j}\left(x ; \partial_{x}\right)}+\underbrace{\tilde{\mathscr{L}}\left(x, t ; \partial_{x}\right)}] u, \\
& \text { the part where the parts where modified } \\
& \text { modified order orders are less than that } \\
& \text { is maximum. of } t^{n_{j}} P_{j} \text {. }
\end{aligned}
$$

$$
\left.u\right|_{t=0}=u_{0}(x) \in H\left(\Omega_{x}\right)
$$

where order $P_{k} \geqq 2,\left(0 \leqq n_{1}<n_{2}<\cdots<n_{k}\right)$.

Let $a_{p_{j}}(x)$ be a coefficient of $\partial_{x_{1}}^{p_{j}}$ of $P_{j}(j=1, \ldots, k)$, then since $P_{k}\left(x ; \partial_{x}\right) \not \equiv 0$, we may assume that $a_{p_{k}} \not \equiv 0$, if necessary transforming the coordinates. Therefore without loss of generality, we assume that $a_{p_{k}} \neq 0$ in a neighborhood of the origin. Let $u(x, t) \sim \sum_{m \geqq 0} u_{m}(x) t^{m} / m$ ! be a formal solution of the problem (5.1)-(5.2), then for $m=\sum_{i} l_{i}\left(n_{i}+1\right), l_{i} \geqq 0$ (integer) we have

$$
u_{m}(x)=Q_{m}\left(x ; \partial_{x}\right) u_{0}(x)+R_{m}\left(x ; \partial_{x}\right) u_{0}(x)
$$

where order $Q_{m}=\frac{m p_{k}}{n_{k}+1}$, order $R_{m}<\frac{m p_{k}}{n_{k}+1}$. From (3.7) we know that $Q_{m}(x ; \zeta)=\sum_{i=1}^{k} \frac{m !}{m \cdot\left(m-n_{i}-1\right) !} P_{i}(x ; \zeta) Q_{m-n_{i}-1}(x ; \zeta)\left(\zeta \in C^{s}\right)$, therefore the coefficient $a_{m}(x)$ of $\partial_{x_{1}}^{m \cdot p_{k} /\left(n_{k}+1\right)}$ of $Q_{m}$ is given by

$$
a_{m}(x)=\sum_{i} \frac{m !}{m \cdot\left(m-n_{i}-1\right) !} a_{p_{i}}(x) a_{m-n_{i}-1}(x) .
$$


Hence

$$
u_{m}(x)=a_{m}(x) \partial_{x_{1}}^{m p_{k} /\left(n_{k}+1\right)} u_{0}(x)+\widetilde{R}_{m}\left(x ; \partial_{x}\right) u_{0}(x),
$$

where order $\widetilde{R}_{m} \leqq m p_{k} /\left(n_{k}+1\right)$ and order of $\widetilde{R}_{m}$ with respect to $x_{1}<m \cdot p_{k} /$ $\left(n_{k}+1\right)$. Without loss of generality we may assume that $a_{p_{j}}(0) \neq 0$ for $j=1,2, \ldots, k$, then by Lemma 5 for infinitely many $m a_{m}(0) \neq 0$. Let us consider the series $\left\{a_{m}(0) ; m=\sum l_{i}\left(n_{i}+1\right)\right\}$ satisfying the asymptotic formula (4.1).

Now let $\left\{a_{n(j)}(0)\right\}_{j=0}^{\infty}$ be a subsequence satisfying lemma 6 , and let

$$
\left.u\right|_{t=0}=\sum_{j=0}^{\infty} e^{\mathrm{i} \theta_{J}}\left(\lambda x_{1}\right)^{n(j) p_{k} /\left(n_{k}+1\right)},
$$

be a Cauchy datum of (5.1), where we choose $\lambda>0$ so that $\left\{x \in \mathbb{C}^{s}\right.$; $\left.\left|\lambda x_{1}\right|<1\right\} \supset \Omega_{x}$. Thus, Cauchy datum belongs to $H\left(\Omega_{x}\right)$.

Substituting (5.2)' into (5.5), it follows that

$$
u_{n(j)}(0)=a_{n(j)}(0)\left\{\frac{n(j) p_{k}}{n_{k}+1}\right\} ! \lambda^{n(j) p_{k} /\left(n_{k}+1\right)} e^{\mathrm{i} \theta_{j}}+f_{n(j)}\left(\theta_{0}, \theta_{1}, \ldots, \theta_{j-1}\right),
$$

where $f_{n(j)}$ is a constant depending only on $\theta_{0}, \ldots, \theta_{j-1}$. We give $\theta_{0}$ arbitrary and if we choose the argument $\theta_{j}(j=1,2, \ldots)$ satisfying

$$
\theta_{j}=\arg \left(f_{n(j)}\right)-\arg \left(a_{n(j)}(0)\right)
$$

we have

$$
\left|u_{n(j)}(0)\right|>\left|a_{n(j)}(0)\right| \cdot\left\{\frac{n(j) p_{k}}{n_{k}+1}\right\} ! \lambda^{(n j) p_{k} /\left(n_{k}+1\right)}
$$

Thus

$$
\sqrt[n(j)]{\left|u_{n(j)}(0)\right| / n(j) !} \geqq \lambda^{\frac{p_{k}}{n_{k}+1}} \cdot \sqrt[n(j)]{\left|a_{n(j)}(0)\right| \cdot\left\{\frac{n(j) p_{k}}{n_{k}+1}\right\} ! / n(j) !} .
$$

Therefore Lemma 6 implies

$$
\sqrt[n(j)]{\left|u_{n(j)}(0)\right| / n(j) !} \longrightarrow+\infty \quad \text { as } j \longrightarrow+\infty
$$

(5.8) shows that the formal solution $\sum_{m \geqq 0} u_{m}(x) t^{m} / m$ ! is not holomorphic in any neighborhood of the origin. 
Finally we note that at a point $x_{0}$ such as $a_{p_{k}}\left(x_{0}\right) \neq 0$, we can construct such a Cauchy datum that there can not exist holomorphic solution in any neighborhood of $\left(x_{0}, 0\right)$. This completes the proof.

Q.E.D.

This argument of the proof is originally used in [1], (see also [2], [5]).

\section{References}

[1] Hasegawa, Y., On the initial-value problems with data on a double characteristic, J. Math. Kyoto Univ. 11 (1971) 357-372.

[2] Miyake, M., On the initial-value problems with data on a characteristic surface for linear systems of first order equations, Publ. R. I. M.S. Kyoto Univ. 8 (1972) 231-264.

[3] - Degenerate parabolic differential equations: Necessity of the wellposedness of the Cauchy problem, to appear in J. Math. Kyoto Univ.

[4] - Degenerate parabolic differential equations: hypoellipticity, in preparation.

[5] Mizohata, S., On kuvalevskian system, to appear in Lispehi Mat. Nauk (in Russian).

[6] The theory of partial differential equations, Camb. Univ. Press, 1973. 
